\title{
Mutational analysis of the MECP2 gene in Japanese patients with Rett syndrome
}

\begin{abstract}
Rett syndrome is a neurodevelopmental disorder observed almost exclusively in girls, and is characterized by autistic tendency, severe mental retardation, stereotyped hand movements, seizures, and acquired microcephaly. Recently, the MECP2 (methyl-CpG-binding protein 2) gene, mapped on chromosome Xq28, was reported to be responsible for Rett syndrome. We performed mutational analysis of the MECP2 gene in 26 Japanese patients with Rett syndrome (who were sporadic cases), and identified disease alleles in 19 patients. The mutations consisted of 12 different types including 3 missense, 3 nonsense, and 6 frameshift mutations. Of these, 8 mutations are novel. Most of these mutations affect the functional domains, methyl$\mathrm{CpG}$ binding domain (MBD), and transcriptional repression domain (TRD), and therefore may critically affect the function of $\mathrm{MeCP} 2$. The disease phenotype of patients with mutations in the MBD tended to be more severe than the phenotype of those with mutations in the TRD. We also identified 2 types of silent mutations and 4 types of missense mutations as benign variants, and these are all novel ones. Most of the nucleotide substitutions involve $\mathrm{C} \rightarrow \mathrm{T}$ transitions at $\mathrm{CpG}$ hotspots. The novel disease alleles and benign variants of the MECP2 gene found in this study should contribute to the establishment of a reliable diagnosis of Rett syndrome.
\end{abstract}

Key words Rett syndrome $\cdot$ Mental retardation $\cdot M E C P 2$ gene $\cdot$ Methyl-CpG-binding protein $\cdot \mathrm{X}$ chromosome dominant $\cdot$ Mutation

K. Amano $\cdot$ K. Yamakawa $(\bowtie)$

Laboratory for Neurogenetics, Brain Science Institute, The Institute of Physical and Chemical Research (RIKEN), 2-1, Hirosawa,

Wako-shi, Saitama 351-0198, Japan

Tel. +81-48-467-9703; Fax +81-48-462-4796

e-mail: yamakawa@brain.riken.go.jp

Y. Nomura $\cdot$ M. Segawa

Segawa Neurological Clinic for Children, Tokyo, Japan

\section{Introduction}

Patients with Rett syndrome (RTT) appear normal in early infancy, but soon begin to show hypotonia, autistic behavior, lack of interlimb coordination, and stereotyped hand movements. Head growth begins to decelerate in late infancy and often shows "acquired" microcephaly when subjects are around 2 years of age. Severe mental retardation, loss of acquired hand use, abnormal respiration, seizure, dystonic posture of limbs, and scoliosis appear agedependently from early childhood. After the initial regression, the disease status is stabilized for decades and patients survive into adulthood. These characteristic clinical features suggest that RTT is a unique neurodevelopmental disorder involving specific neurons and neural systems at a certain age period in the course of development of the brain. The exclusive involvement of females in RTT is explained by X-linked dominant inheritance, with lethality in hemizygous males. The disease frequency is estimated to be 1:10,000-15,000 female births (Hagberg 1995). The majority of cases are sporadic, but rare familial cases with patients whose mothers have skewed $\mathrm{X}$ chromosome inactivation have been reported. Studies of these familial cases have indicated that Xq28 is the candidate region for RTT (Xiang et al. 1998; Sirianni et al. 1998). Recently, the MECP2 gene encoding methyl-CpG-binding protein 2 (MeCP2), which maps to $\mathrm{Xq} 28$, was reported to be responsible for RTT (Amir et al. 1999). MeCP2 contains both a methyl$\mathrm{CpG}$ binding domain (MBD) and a transcriptional repression domain (TRD), both of which mediate gene silencing through their selective binding to methylated $\mathrm{CpG}$ dinucleotides in the genome (Nan et al. 1998; Jones et al. 1998).

It is important to ascertain whether the $M E C P 2$ gene also plays a major role in Japanese patients with RTT. Here, we report the result of mutational analysis for the MECP2 gene in Japanese patients, and the identification of disease alleles at a higher ratio than that reported in previous studies (Amir et al. 1999; Wan et al. 1999). 


\section{Patients and methods}

\section{Subjects}

This study encompassed 26 Japanese patients (sporadic cases) who had been clinically diagnosed with RTT. For 9 of these patients, we obtained samples from their parents and sisters who have not shown signs of RTT clinically. Each participating subject or an adult responsible for the subject signed an informed consent form, as approved by the Institutional Review Board at the Brain Science Institute, The Institute of Physical and Chemical Research (RIKEN).

\section{Mutational analysis}

Genomic DNA samples were extracted from Epstein-Barr virus-transformed lymphoblastoid cell lines of the patients and their family members, with the Qiagen genomic-tip system (Qiagen, Tokyo, Japan). Three exons of the MECP2 gene (Amir et al. 1999) were amplified by the polymerase chain reaction (PCR), using Pyrobest DNA polymerase (Takara, Tokyo, Japan). The nucleotide sequences for the forward and reverse primers used for exon 1 were 5'-GGTGA CAAGGCTTGTGATAG-3' and 5'AGATGGCCAAACCAGGACAT- $3^{\prime}$, and the primers for exon 2 were $5^{\prime}$-TGGCATGTTCTCTGTGATACT-3' and 5'-CTGCTC CATGAGGGATCCT-3'. The sets of PCR primer for exon 3 were described previously (Amir et al. 1999). We performed PCR amplification, using 50-100 ng of genomic DNA in a $25-\mu 1$ volume containing $0.3 \mu \mathrm{M}$ of each primer, $1 \times$ Pyrobest buffer (Takara), $0.2 \mathrm{mM}$ dNTPs, and 2.5U Pyrobest DNA polymerase (Takara). PCR conditions were: initial denaturation at $95^{\circ} \mathrm{C}$ for $5 \mathrm{~min}$, followed by 35 cycles of denaturation at $95^{\circ} \mathrm{C}$, annealing at $60^{\circ} \mathrm{C}$, and extension at $72^{\circ} \mathrm{C}$ for $1 \mathrm{~min}$ each. Amplified PCR products were purified with an ArrayIt PCR purification kit (TeleChem International, Sunnyvale, CA, USA) and sequenced directly with a BigDye terminator cycle sequencing kit and ABI PRISM 3700 DNA sequencing system (PE Applied Biosystems, Tokyo, Japan). The existence or prevalence of each mutation in the general population was tested using DNA samples from 107 normal Japanese control individuals (28 males and 79 females).

\section{Results}

\section{Mutational analyses of RTT patients}

We searched for sequence variations in the $M E C P 2$ gene, using seven sets of PCR primers, covering the coding region as well as the splice junctions, in the 26 Japanese patients with RTT (sporadic cases) and 16 of their normal family members. The results are summarized in Table 1, and several representative electropherograms of mutations are shown in Fig. 1.

We found mutations that are predicted to be responsible for RTT in 19 patients (73\%), and 7 patients did not show any mutations responsible for disease in the coding region. The mutations responsible for disease were categorized into 12 types, including 3 nonsense mutations (R168X, R270X, and R294X), 6 frameshift mutations (201GlyFS-235X, 269AlaFS-288X, and micro deletions of $34 \mathrm{bp}, 388 \mathrm{bp}$, $259 \mathrm{bp}$, and $154 \mathrm{bp}$ ), and 3 missense mutations (T158M, $\mathrm{P} 302 \mathrm{H}$, and $\mathrm{R} 306 \mathrm{C})$. The $\mathrm{R} 168 \mathrm{X}$ nonsense mutation is a heterozygous $\mathrm{C} \rightarrow \mathrm{T}$ mutation $(576 \mathrm{C} \rightarrow \mathrm{T})$, introducing a stop codon in the place of arginine, this mutation was found in four patients (Seg-005, 010, 012, and 033). The other two nonsense mutations (R270X in patient Seg-006; R294X in patients Seg-004 and 051) were also a heterozygous $\mathrm{C} \rightarrow \mathrm{T}$ mutation. In patient Seg-013, a heterozygous insertion, 676insG, at codon 201 shifts the reading frame and introduces a stop codon after 34 amino acids (201GlyFS-235X). In patient Seg-017, a heterozygous deletion, 880delG, at codon 269 introduces a stop codon after 19 amino acids (269AlaFS-288X). Four other frameshift mutations, which were polynucleotide deletions, including $34 \mathrm{bp}$ (nucleotides 859 to 892 in patient Seg-020), 388 bp (nucleotides 922 to 1309, or nucleotides 923 to 1310 in patient Seg-018), 259 bp (nucleotides 988 to 1246 in patient Seg-036), and $154 \mathrm{bp}$ (nucleotides 1110 to 1263 , or nucleotides 1111 to 1264 , or nucleotides 1112 to 1265 in patient Seg-009), introduced a stop codon after 15, 69, 17, and 11 amino acids, respectively. We could not determine the exact position of the deletion precisely in patients Seg-018 and 009 because of matched nucleotides at both ends of the deletions. Three missense mutations (T158M in Seg-019, 043 and 044; P302H in Seg040; R306C in Seg-011 and 015) were observed only in patients, and were not observed in unaffected family members or in the 107 independent normal controls; therefore, these were assumed to be responsible for RTT.

Sequence variants that may not be responsible for the disease phenotype include four types of missense (A201V, G232A, P251L, and P376S) and two types of silent nucleotide changes (P56P and T442T). The G232A missense mutations found in patients Seg-005, 010, and 012 were observed hemizygously or heterozygously in 10 (1 male and 9 female) of the 107 control individuals (10 of $186 \mathrm{X}$ chromosomes; $5.4 \%$ ), and these mutations were considered to constitute a non-disease allele. The allele of P251L in patient Seg-009 was also found in the patient's father, Seg057, and the allele did not appear in the 107 normal controls. In addition, the 154-bp deletion appeared as a disease allele in this patient. These findings indicate that $\mathrm{P} 251 \mathrm{~L}$ is a rare, but non-disease, allele. A mutation (A201V), which was found in patient Seg-030, and was also found in her normal mother and in one female normal control individual $(0.5 \%)$, was considered to be a non-disease allele. P376S mutations were observed only in two normal family members (Seg-037 and 039). Two silent mutations (P56P and $\mathrm{T} 442 \mathrm{~T}$ ) were also found (Table 1).

\section{Genotype and phenotype correlation}

The locus and type of disease mutations in the MECP2 gene observed in the 19 patients with RTT were examined in 
Patients-013

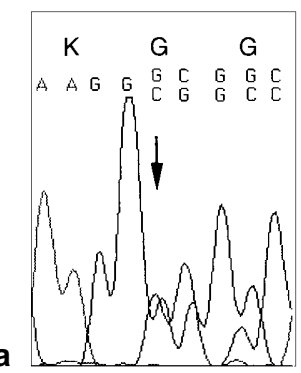

009

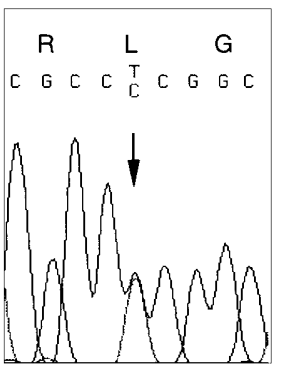

020

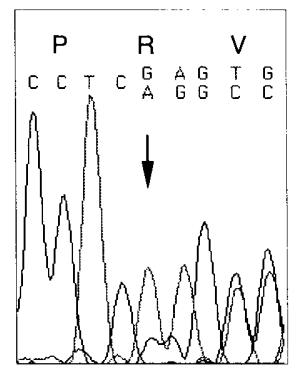

006

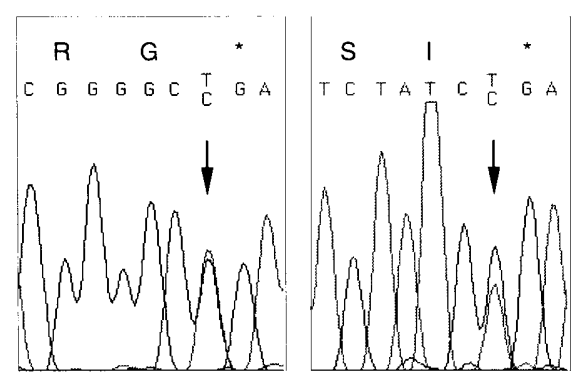

040

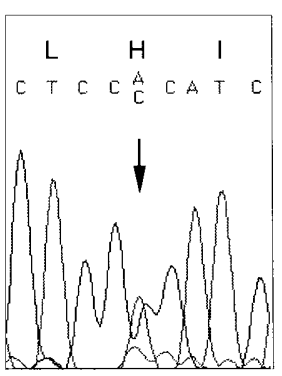

Controls

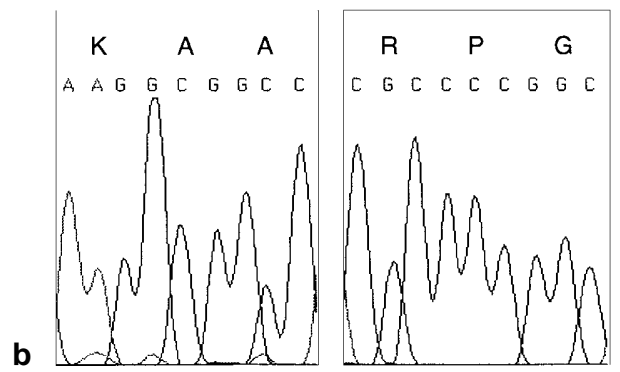

Fig. 1a,b. Electropherograms of DNA sequencing for six mutations of the $M E C P 2$ gene found in patients with Rett syndrome (RTT) and control individuals. a Patients with RTT; b control individuals. Arrows

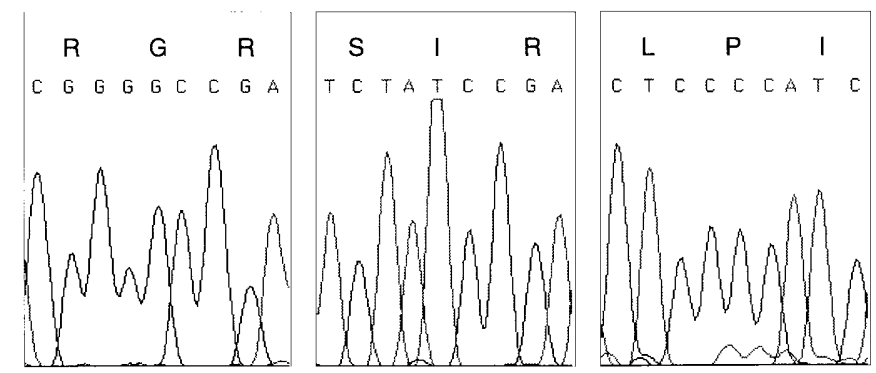

in a indicate positions of nucleotide changes. Predicted amino acids are also indicated. Asterisk, Stop codon relation to the clinical characteristics of each patient (Table 2 ), including markers evaluating the development and particular symptoms of RTT. Of the markers evaluating development, the ages at which head control, crawling, and walking occurred were evaluated, and the age at which the patient first spoke a word was assessed. In regard to the particular symptoms of RTT, the ages at onset of stereotyped hand movement, epilepsy, and respiratory abnormality were evaluated.

Delay in head control was observed only in a patient with the T158M mutation (one of three patients with this mutation). Crawling was impossible or abnormal in pattern in all patients. The patients who did not crawl had the T158M mutation (two of three patients), the R168X mutation (one of four patients), and the R306C mutation (one of two patients). Two other patients with R168X could crawl, but the age at first crawling was abnormally delayed. As for walking, patients with T158M (two of three patients), R168X (three of four patients), 201GlyFS-235X, and the 388-bp deletion did not walk. The patient with $\mathrm{P} 302 \mathrm{H}$ walked after the age of 5 years and 4 months. Patients with the 34-bp deletion mutation, the R294X mutation (one of two patients) and the R306C mutation (one of two patients) first walked at ages ranging from 1 year, 6 months to 2 years, 1 month.

The ability to speak is not shown in most patients with RTT. Very few patients can speak, and then only a few simple words are possible. The patients with T158M, R168X (two of four patients), 201GlyFS-235X, the 34-bp deletion, $\mathrm{R} 270 \mathrm{X}, \mathrm{P} 302 \mathrm{H}$, and R306C have never spoken a word, while patients with the mutations 269AlaFS-288X, the 388bp deletion, R294X (one of two patients), the 259-bp deletion, and the 154-bp deletion spoke simple words before age 2 years. Thus, the results in regard to speaking were similar in tendency to the results for crawling and walking.

Hand stereotypy is the pathognomonic symptom of RTT. The patients with the T158M mutation (two of three patients), the R168X mutation (three of four patients), and the 34-bp deletion developed stereotyped hand movements before age 1 year, 6 months. The patients with the 269AlaFS-288X and R294X mutations started to show stereotypy after age 3 years, the rest of the 19 RTT patients with disease mutations in the $M E C P 2$ gene started to show stereotypy between age 1 year, 6 months and age 3 years.

Epilepsy is often seen in RTT. The patients who did not show epilepsy were those with R270X, R294X, and the 259bp deletion. One patient with R168X had not had clinical seizures, but the EEG was markedly abnormal. Abnormal respiratory patterns were observed in all patients except for those with the T158M mutation (two of three patients), R168X (one of four patients), 201GlyFS-235X, P302H, $259 \mathrm{bp}$ deletion, R306C (one of two patients) and the 154-bp deletion.

In summary, those patients with the T158M, R168X, and 201GlyFS-235X mutations were severely affected in regard 
Table 1. Summary of disease mutations and benign variants of the $M E C P 2$ gene found in RTT patients and their family members

\begin{tabular}{|c|c|c|c|c|}
\hline Patients & Nonsense mutation & Frameshift mutation & Missense mutation & Silent mutation \\
\hline Seg-001 & - & - & - & - \\
\hline Seg-004 & $R 294 X(954 C \rightarrow T)$ & - & - & $\mathrm{T} 442 \mathrm{~T}(1400 \mathrm{C} \rightarrow \mathrm{T}) *$ \\
\hline Seg-005 & $R 168 X(576 C \rightarrow T)^{*}$ & - & G232A $(769 \mathrm{G} \rightarrow \mathrm{C})$ & - \\
\hline Seg-006 & $R 270 X(882 C \rightarrow T)^{*}$ & - & - & - \\
\hline Seg-009 & - & Deletion $(154 b p)$ & P251L $(826 \mathrm{C} \rightarrow \mathrm{T})$ & - \\
\hline Seg-010 & $R 168 X(576 C \rightarrow T)^{*}$ & - & $\mathrm{G} 232 \mathrm{~A}(769 \mathrm{G} \rightarrow \mathrm{C})$ & - \\
\hline Seg-011 & - & - & $R 306 C(990 C \rightarrow T)^{*}$ & - \\
\hline Seg-012 & $R 168 X(576 C \rightarrow T)^{*}$ & - & G232A $(769 \mathrm{G} \rightarrow \mathrm{C})$ & - \\
\hline Seg-013 & - & $201 G l y F S-235 X(676 i n s G)$ & - + r n & - \\
\hline Seg-015 & - & - & $R 306 C(990 C \rightarrow T) *$ & - \\
\hline Seg-016 & - & - & - & - \\
\hline Seg-017 & - & 269 AlaFS-288X (880delG) & - & - \\
\hline Seg-018 & - & Deletion (388bp) & - & - \\
\hline Seg-019 & - & - & $T 158 M(547 C \rightarrow T)^{*}$ & - \\
\hline Seg-020 & - & Deletion (34bp) & - & - \\
\hline Seg-021 & - & - & - & - \\
\hline Seg-025 & - & - & - & - \\
\hline Seg-030 & - & - & A201V $(676 \mathrm{C} \rightarrow \mathrm{T})^{*}$ & - \\
\hline Seg-033 & $R 168 X(576 C \rightarrow T)^{*}$ & - & - & - \\
\hline Seg-036 & - & Deletion (259 bp) & - & - \\
\hline Seg-040 & - & - & $P 302 H(979 C \rightarrow A)$ & - \\
\hline Seg-043 & - & - & $T 158 M(547 C \rightarrow T)^{*}$ & - \\
\hline Seg-044 & - & - & $T 158 M(547 C \rightarrow T) *$ & - \\
\hline Seg-045 & - & - & - & - \\
\hline Seg-049 & - & - & - & $\mathrm{P} 56 \mathrm{P}(242 \mathrm{C} \rightarrow \mathrm{T})^{*}$ \\
\hline Seg-051 & $R 294 X(954 C \rightarrow T)^{*}$ & - & - & - \\
\hline \multicolumn{5}{|c|}{ Normal family members } \\
\hline Seg-057 (009F) & - & - & P251L $(826 \mathrm{C} \rightarrow \mathrm{T})$ & - \\
\hline Seg-058 (009M) & - & - & - & - \\
\hline Seg-053 (016F) & - & - & - & - \\
\hline Seg-054 (016M) & - & - & - & $\mathrm{T} 442 \mathrm{~T}(1400 \mathrm{C} \rightarrow \mathrm{T})^{*}$ \\
\hline Seg-031 (030F) & - & - & - & - \\
\hline Seg-032 (030M) & - & - & A201V $(676 \mathrm{C} \rightarrow \mathrm{T})^{*}$ & - \\
\hline Seg-034 (033F) & - & - & - & - \\
\hline Seg-035 (033M) & - & - & - & - \\
\hline Seg-037 (036F) & - & - & P376S $(1200 \mathrm{C} \rightarrow \mathrm{T})$ & - \\
\hline Seg-038 (036M) & - & - & - & - \\
\hline Seg-039 (036S) & - & - & P376S $(1200 \mathrm{C} \rightarrow \mathrm{T})$ & - \\
\hline Seg-041 (040F) & - & - & - & - \\
\hline Seg-042 (040M) & - & - & - & - \\
\hline Seg-048 (043M) & - & - & - & - \\
\hline Seg-050 (049M) & - & - & - & - \\
\hline Seg-052 (051M) & - & - & - & - \\
\hline
\end{tabular}

Nucleotide and amino acid changes are numbered according to the MECP2 sequences registered in GenBank (accession number, X99686). Mutations that are considered to be disease alleles are italic. In information for normal family members, $\mathrm{F}$ indicates father, $\mathrm{M}$ indicates mother, and S indicates sister. For example, Seg-057 (009F) means that member Seg-057 is the father of patient Seg-009. Mutations marked with asterisks indicate that $\mathrm{C} \rightarrow \mathrm{T}$ transitions occurred at $\mathrm{CpG}$ hot spots

RTT, Rett syndrome

to crawling, walking, and language ability, and association with epilepsy was frequent, while the other patients with mutations including R294X, 269AlaFS-288X, and the 259bp deletion had mild clinical symptoms.

\section{Discussion}

Here we reported the results of mutational analysis for the $M E C P 2$ gene in 26 Japanese RTT patients. In these 26 patients, we identified 19 disease mutations $(73 \%)$. The rate of mutation detection in our samples is higher than that in previous reports - such as the approximately $50 \%$ detection rate reported by Wan et al. (1999) — and it confirms the major role of the $M E C P 2$ gene in the etiology of Japanese RTT patients.

Among 25 nucleotide substitutions found in the patients and their family members, 21 were $\mathrm{C} \rightarrow \mathrm{T}$ transitions. Of these transitions, 16 were at $\mathrm{CpG}$ hotspots, and the remaining 5 were at $\mathrm{CpC}$ sequences. This high ratio of mutations in $\mathrm{CpG}$ hotspots may be responsible for the findings of common mutations, including T158M, R168X, and R306C, reported in previous studies (Amir et al. 1999; Wan et al. 1999) (Fig. 2). The 269AlaFS-288X single nucleotide deletion mutation is also common; however, it is too early to discuss a tendency for the deletion of a specific nucleotide, 880G. Unlike results in previous studies, we identified several patients with microdeletions, including deletions of 34 bp, 388 bp, 259 bp, and 154 bp (Fig. 2). We are not sure 
Table 2. Phenotypes of RTT patients - ages of acquisition of each milestone and of appearance of characteristic symptoms

\begin{tabular}{|c|c|c|c|c|c|c|c|c|}
\hline Patient & $\begin{array}{l}\text { Type of } \\
\text { disease mutation }\end{array}$ & $\begin{array}{l}\text { Head } \\
\text { control }\end{array}$ & Crawling $^{\mathrm{a}}$ & Walking & Speaking a word & Hand stereotypy & Epilepsy & $\begin{array}{l}\text { Abnormal } \\
\text { respiration }\end{array}$ \\
\hline \multicolumn{9}{|c|}{ Mutations in or close to MBD } \\
\hline Seg-019 & $\mathrm{T} 158 \mathrm{M}$ & 4 Months & 1 Year & 1 Year 5 Months & - & 1 Year 4 Months & 3 Years 3 Months & - \\
\hline Seg-043 & $\mathrm{T} 158 \mathrm{M}$ & $>4$ Months & - & - & - & 1 Year 6 Months & 3 Years 6 Months & - \\
\hline Seg-044 & $\mathrm{T} 158 \mathrm{M}$ & 5-6 Months & - & - & - & 2 Years 6 Months & 2 Years 1 Month & 3 Years 2 Months \\
\hline Seg-005 & $\mathrm{R} 168 \mathrm{X}$ & 4 Months & 1 Year & 1 Year 5 Months & $>1$ Year? & 2 Years 3 Months & 2 Years 11 Months & 2 Years 3 Months \\
\hline Seg-010 & R168X & 3 Months & 2 Years & - & 2 Years & 1 Year 6 Months & - & - \\
\hline Seg-012 & R168X & 3 Months & - & - & - & 1 Year 4 Months & 10 Months & $\pm(2-3 \text { Years })^{b}$ \\
\hline Seg-033 & R168X & 4 Months & 3 Years 10 Months & - & - & 10 Months & 3 Years 9 Months & 2 Years 2 Months \\
\hline Seg-013 & 201GlyFS-235X & 3 Months & 8 Months & - & - & 2 Years & 7 Years 4 Months & - \\
\hline \multicolumn{9}{|c|}{ Mutations in or close to TRD } \\
\hline Seg-020 & Deletion (34 bp) & 3 Months & 8 Months & 1 Year 11 Months & - & 1 Year 3 Months & 1 Year 11 Months & 3 Years 4 Months \\
\hline Seg-017 & 269AlaFS-288X & 3 Months & 7 Months & 11 Months & 1 Year 3 Months & 4 Years 6 Months & 3 Years 4 Months & 4 Years 2 Months \\
\hline Seg-006 & $\mathrm{R} 270 \mathrm{X}$ & 3-4 Months & 9 Months & 1 Year 2 Months & - & 2 Years 1 Month & - & 3 Years 6 Months \\
\hline Seg-018 & Deletion (388bp) & 4 Months & 10 Months & - & 1 Year 2 Months & 2 Years 4 Months & 5 Years & 2 Years 10 Months \\
\hline Seg-004 & R294X & 3 Months & 9 Months & 1 Year 3 Months & 1 Year & 9-10 Years & - & 8 Years \\
\hline Seg-051 & R294X & 4 Months & $<1$ Year & 2 Years 1 Month & 2 Years & $\geq 3$ Years & - & 4 Years 3 Months \\
\hline Seg-040 & $\mathrm{P} 302 \mathrm{H}$ & 4 Months & 2 Years & 5 Years 4 Months & - & 2 Years & 4 Years 3 Months & - \\
\hline Seg-036 & Deletion (259bp) & 3 Months & 8 Months & 1 Year 3 Months & 1 Year 6 Months & 2 Years 6 Months & - & - \\
\hline Seg-011 & $\mathrm{R} 306 \mathrm{C}$ & 4 Months & 11 Months & 1 Year 3-4 Months & - & 2 Years $4-5$ Months & 5 Years 6 Months & 2 Years 3-4 Months \\
\hline Seg-015 & $\mathrm{R} 306 \mathrm{C}$ & 4 Months & - & 1 Year 6 Months & - & 2 Years 4 Months & 2 Years & - \\
\hline Seg-009 & Deletion (154bp) & 3-4 Months & 1 Year & 1 Year 5 Months & 11 Months & 3 Years & 2 Years 4 Months & - \\
\hline
\end{tabular}

-, Milestone never acquired, or symptom never occurred

MBD, Methyl-CpG binding domain; TRD, transcriptional repression domain

${ }^{a}$ Even in those patients who could crawl, the posture and pattern of crawling were markedly abnormal

${ }^{\mathrm{b}}$ Diagnosis not confirmed

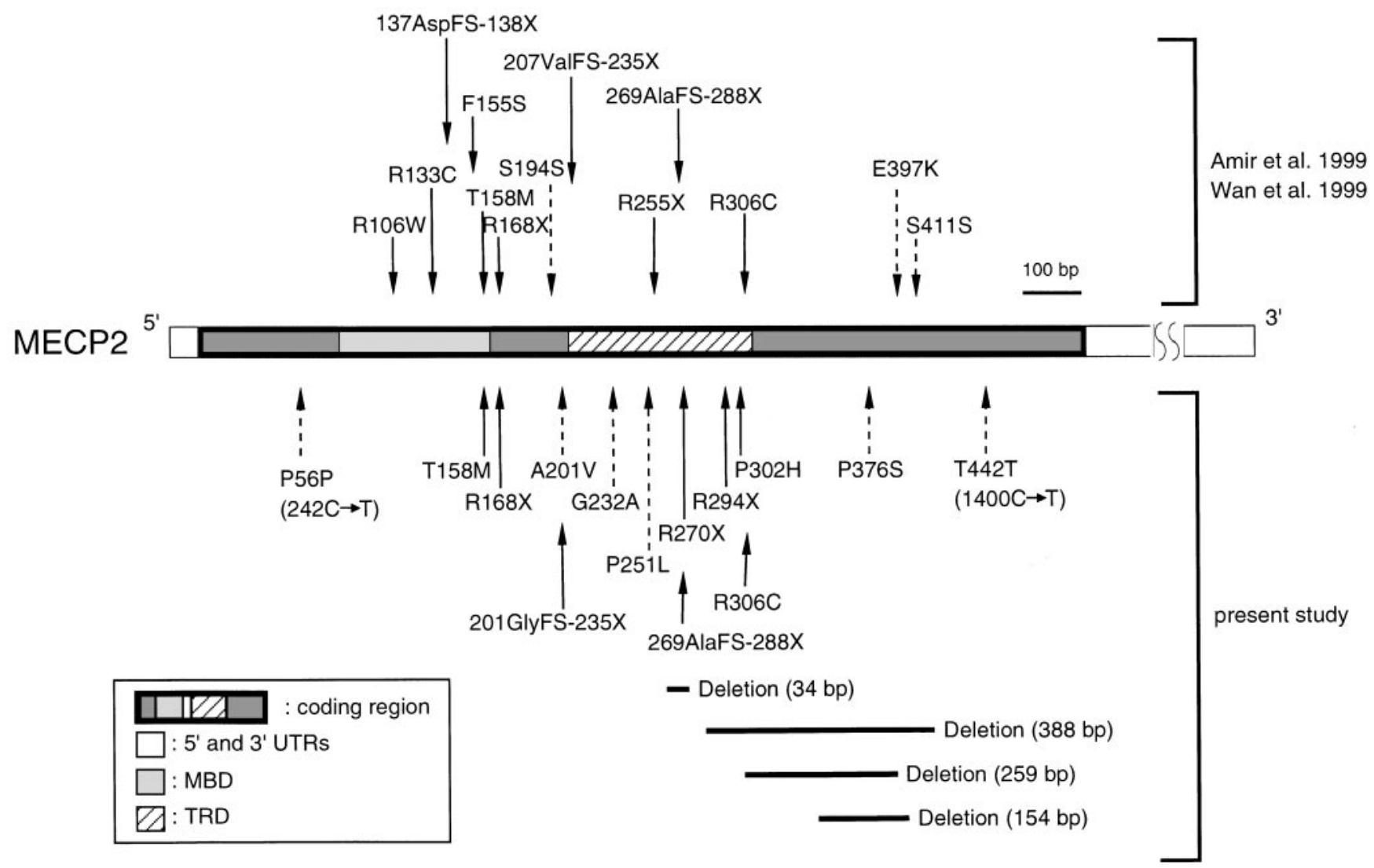

Fig. 2. Localization of RTT mutations found in the coding regions of the MECP2 gene. Upper panel shows the mutations found in previous studies (Amir et al. 1999; Wan et al. 1999). The lower panel shows mutations found in the present study. Arrows with solid lines indicate disease alleles. Arrows with dashed lines indicate benign variants. UTR, Untranslated region; $M B D$, methyl-CpG binding domain (amino acids nos. 78-160); TRD, transcriptional repression domain (amino acids nos. 207-311) 
whether these microdeletions are characteristic of Japanese mutations, but these findings could also contribute to the establishment of more reliable diagnosis of RTT patients.

Most of the disease mutations affected the functional domains of the $M E C P 2$ gene (Fig. 2). T158M mutations occurred in the methyl-CpG binding domain (MBD). R168X and 201GlyFS-235X mutations occurred between the MBD and TRD domains and deleted the whole TRD domain. The 34-bp deletion, 269AlaFS-288X, R270X, the 388-bp deletion, R294X, and the 259-bp deletion mutations removed parts of the TRD domain. The $\mathrm{P} 302 \mathrm{H}$ and $\mathrm{R} 306 \mathrm{C}$ mutations occurred in the TRD domain. Among the mutations reported in the present study, as well as in previous studies (Amir et al. 1999; Wan et al. 1999), only the mutation of the 154-bp microdeletion does not affect MBD nor TRD functional domains (Fig. 2). Although we cannot completely exclude the possibility that this microdeletion is a rare polymorphism and is not responsible for the RTT phenotype, the finding that the 107 normal controls did not have this deletion, and the finding that this microdeletion results in the lack of a large $\mathrm{C}$-terminal portion of the $\mathrm{MeCP} 2$ protein, suggest that the $\mathrm{C}$-terminal downstream of the functional domains is also important or responsible for the disease phenotype.

A201V, G232A, P251L, and P376S are novel missense mutations found in our study. All these mutations were judged as benign variants or, at least, as not responsible for the disease phenotype of RTT, as described in "Results". However, these amino acid changes, especially those occurring in the TRD domain, may alter the functional property of the protein. Even if these mutations do not lead directly to the phenotype of RTT, it is still possible that these variants may result in phenotypic variability or susceptibility to other diseases. Although we are not sure at present whether or not these polymorphisms appear only in Japanese, it is also possible that these amino acid changes, especially the G232A mutation, which occurs at a frequency of $5.4 \%$ in the normal Japanese population, could be responsible for Japanese-specific characteristics.

The conclusion proposed by Wan et al. (1999) previously suggested that the $\mathrm{X}$-chromosome inactivation (XCI) pattern might be the major determinant of the RTT phenotype. However, our study described here suggests some correlation between types of mutations and disease phenotypes in RTT patients. The patients with the T158M, R168X, and 201GlyFS-235X mutations that reside in or are close to the MBD domain were severely affected in terms of crawling and walking, while patients with other mutations that reside or affect the TRD domain showed milder phenotypes (Fig. 2; Table 2). In addition, the patients with the MBD-related mutations tended to show more severe verbal abnormalities and a rather frequent association with epilepsy. These results may suggest that the retained TRD domains, even though they are partial, do function, and lead to milder phenotypes.

In a clinical evaluation of 38 patients with Rett syndrome, Segawa et al. (1998) suggested that the ages at which patients started to crawl and to walk were correlated to the mental activities evaluated by language ability. It was also shown that the age at which the patients developed the stereotyped movement had no correlation to language ability (Segawa et al. 1998). Head control, crawling, and walking are representative features indicating the development of postural augmentation and locomotion. Thus, our present data suggest that patients with the T158M, R168X, and 201GlyFS-235X mutations had severe abnormalities in the postural augmentation system and in locomotion, and that these mutations also caused marked disturbance in the acquisition of language. Most of the mutation loci which induced marked abnormalities in locomotion and language acquisition also induced development of the stereotyped hand movement at earlier ages, but were not necessarily related to the respiratory abnormalities. The study of genotype-phenotype correlation requires precise analyses in a larger number of patients to determine correlations with various types of mutations, and also requires functional analyses of $\mathrm{MeCP} 2$, including studies of animal models. XCI is one of the potential mechanisms to be clarified further.

The results described here should contribute to the establishment of a reliable diagnosis for RTT patients and may help to elucidate the pathophysiology and etiology of RTT.

Acknowledgments We thank the patients with RTT, and their parents and families for their participation in this study. We also wish to thank Dr. Naohisa Watanabe and Dr. Jyoji Inazawa for providing us with the DNA of normal control individuals, and Dr. Subramaniam Ganesh for helpful discussion.

\section{References}

Amir RE, Van den Veyver IB, Wan M, Tran CQ, Francke U, Zoghbi HY (1999) Rett syndrome is caused by mutations in X-linked $M E C P 2$, encoding methyl-CpG-binding protein 2. Nat Genet 23:185-188

Hagberg B (1995) Rett syndrome: clinical peculiarities and biological mysteries. Acta Paediatr 84:971-976

Jones PL, Veenstra GJC, Wade PA, Vermaak D, Kass SU, Landsberger N, Strouboulis J, Wolffe AP (1998) Methylated DNA and $\mathrm{MeCP} 2$ recruit histone deacetylase to repress transcription. Nat Genet 19:187-191

Nan X, Ng HH, Johnson CA, Laherty CD, Turner BM, Eisenman RN, Bird A (1998) Transcriptional repression by the methyl-CpGbinding protein $\mathrm{MeCP} 2$ involves a histone deacetylase complex. Nature 393:386-389

Segawa M, Takano M, Shimohira M, Tanaka R, Hachimori K, Nomura Y (1998) Locomotion in late infancy and development of higher cortical function at later ages. In: Perat MV (ed) New developments in child neurology. Monduzzi Editore, International Proceedings Division, Bologna, pp 27-30

Sirianni N, Naidu S, Pereira J, Pillotto RF, Hoffman EP (1998) Rett syndrome: confirmation of X-linked dominant inheritance, and localization of the gene to Xq28. Am J Hum Genet 63:1552-1558

Wan M, Lee SSJ, Zhang X, Houwink-Manville I, Song HR, Amir RE, Budden S, Naidu S, Pereira JLP, Lo IFM, Zoghbi HY, Schanen NC, Francke U (1999) Rett syndrome and beyond: recurrent spontaneous and familial MECP2 mutations at $\mathrm{CpG}$ hotspots. Am J Hum Genet 65:1520-1529

Xiang F, Zhang Z, Clarke A, Joseluiz P, Sakkubai N, Sarojini B, Delozier-Blanchet CD, Hansmann I, Edstrom L, Anvret M (1998) Chromosome mapping of Rett syndrome: a likely candidate region on the telomere of Xq. J Med Genet 35:297-300 